\title{
Identification in rats of a programming window for reproductive tract masculinization, disruption of which leads to hypospadias and cryptorchidism
}

\author{
Michelle Welsh, Philippa T.K. Saunders, Mark Fisken, Hayley M. Scott, \\ Gary R. Hutchison, Lee B. Smith, and Richard M. Sharpe
}

MRC Human Reproductive Sciences Unit, Centre for Reproductive Biology, The Queen's Medical Research Institute, Edinburgh, United Kingdom.

\begin{abstract}
Becoming a phenotypic male is ultimately determined by androgen-induced masculinization. Disorders of fetal masculinization, resulting in hypospadias or cryptorchidism, are common, but their cause remains unclear. Together with the adult-onset disorders low sperm count and testicular cancer, they can constitute a testicular dysgenesis syndrome (TDS). Although masculinization is well studied, no unifying concept explains normal male reproductive development and its abnormalities, including TDS. We exposed rat fetuses to either anti-androgens or androgens and showed that masculinization of all reproductive tract tissues was programmed by androgen action during a common fetal programming window. This preceded morphological differentiation, when androgen action was, surprisingly, unnecessary. Only within the programming window did blocking androgen action induce hypospadias and cryptorchidism and altered penile length in male rats, all of which correlated with anogenital distance (AGD). Androgen-driven masculinization of females was also confined to the same programming window. This work has identified in rats a common programming window in which androgen action is essential for normal reproductive tract masculinization and has highlighted that measuring AGD in neonatal humans could provide a noninvasive method to predict neonatal and adult reproductive disorders. Based on the timings in rats, we believe the programming window in humans is likely to be 8-14 weeks of gestation.
\end{abstract}

\section{Introduction}

In mammals, the reproductive system is initially indistinguishable between the two sexes but is predetermined to develop along the female pathway. To become a male requires modification of this process, involving a chain of events initiated by activation of the Sry gene, resulting in testis formation (1-3). This is the first step along the male pathway but does not itself result in a male phenotype. Rather, body-wide "masculinization" is driven by hormones produced by the fetal testis, primarily testosterone (4). Impaired fetal androgen action interferes with masculinization and can result in disorders of sexual differentiation $(5,6)$ or phenotypic sex reversal, although this is rare. In contrast, milder disorders of human male reproductive development, cryptorchidism (7) and hypospadias (8), are among the most common congenital disorders (occurring in $2.4 \%-9 \%$ and $0.4 \%-1 \%$ of boys at birth, respectively; refs. 9,10 ) and are related to, and are risk factors for, important disorders that emerge in young adulthood, such as low sperm counts and testicular cancer (11). These disorders are hypothesized to constitute a testicular dysgenesis syndrome (TDS) with a proposed common origin in fetal life (11-13); TDS disorders are common and/or increasing progressively in incidence $(7,8,11)$. Deficient testosterone production/action is postulated to underlie TDS disorders $(11,14)$, but this is unproven, as direct determination of

Nonstandard abbreviations used: AGD, anogenital distance; AR, androgen receptor; EW, early window; FW, full window; LW, late window; MW, middle window; TDS, testicular dysgenesis syndrome.

Conflict of interest: The authors have declared that no conflict of interest exists. Citation for this article: J. Clin. Invest. 118:1479-1490 (2008). doi:10.1172/JCI34241. fetal androgen exposure at this age in humans is difficult and TDS disorders are only identified postnatally. One way to test this is by using animal models in which androgen action is inhibited by exposure to pure anti-androgens such as flutamide $(15,16)$.

The fetal testes produce testosterone from 8 to 37 weeks' gestation in humans (17) (E15.5-E21.5 in rats; ref. 18), and during this period, masculinization of the fetal reproductive tract occurs, resulting in differentiation of the internal (epididymis, vas deferens, seminal vesicles, prostate) and external (penis, scrotum, perineum) genitalia (19). Masculinization of these structures does not occur synchronously; the prostate forms at gestation weeks 10-13 (20) (E18.5-E19.5, rat; ref. 21), the penis at weeks 11-13 (20) (E17.5, rat; ref. 22), and seminal vesicles at weeks 14-16 (23) (E19.5, rat; ref. 21). Other androgen-dependent events, such as testis descent into the scrotum, occur much later (human, 27-35 weeks; refs. 24-26; rat, postnatal week 3; ref. 26). Furthermore, masculinization of the brain is a late gestational event in primates, in contrast to the earlier masculinization of the genitalia (27). As a result of such observations, it has been presumed that masculinization occurs over a broad period of fetal life coincident with the broad period of testosterone production in both humans and rats $(17,18)$. The current belief is that androgens induce and complete masculinization of the reproductive tract during the time when morphological differentiation can be observed in these tissues, i.e., late in fetal life for rat reproductive tissues such as the prostate and penis. As a result, researchers investigating the mechanistic basis for androgen-driven masculinization have focused on this period in fetal life (28-30). However, our recent studies investigating wolffian duct development (31) caused us to question this 

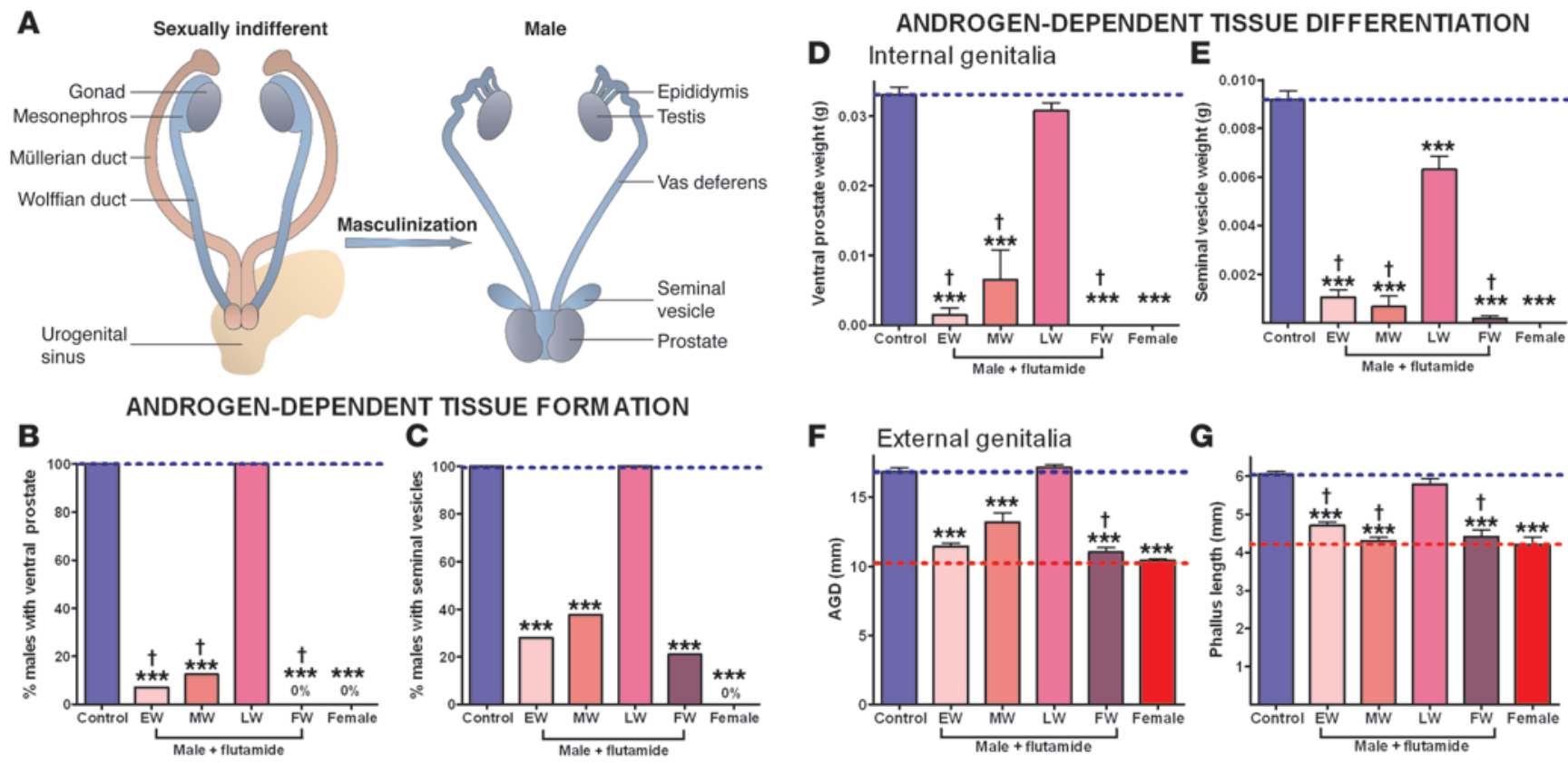

\section{Figure 1}

Identification of the early programming window for masculinization of the reproductive tract in rats. Internal and external genitalia from male rats exposed in utero to flutamide in EW (E15.5-E17.5), MW (E17.5-E19.5), LW (E19.5-E21.5), or FW (E15.5-E21.5) were examined at P25. (A) Schematic diagram of normal masculinization of the indifferent reproductive tissues into their derivative tissues. EW or MW flutamide exposure prevents prostate (B) and seminal vesicle (C) formation in almost all males at P25, while LW flutamide does not prevent prostate or seminal vesicle formation in any males. Ventral prostates (D) and seminal vesicles (E) are significantly smaller in the few EW and MW flutamide-exposed males that had any tissue present. AGD $(\mathbf{F})$ and phallus length $(\mathbf{G})$ are reduced to near female levels in EW and MW flutamide-exposed males at P25. Values are mean \pm SEM $\left(n=8-28\right.$ rats from 2-5 litters per treatment group). ${ }^{\dagger} P>0.05$ compared with female values; ${ }^{* * *} P<0.001$ compared with control male values. Blue dotted line highlights mean male level; red dotted line highlights mean control female level.

belief and instead highlighted the potential that androgens could "preprogram" masculinization before morphological changes are actually observed. If true, this would place the critical window of androgen action much earlier than previously assumed; this could offer some explanation for why so little advance has been made in elucidating the mechanisms underlying androgen-driven masculinization or its associated disorders.

We therefore hypothesized that androgen-driven masculinization, involving morphological differentiation of the various reproductive tract tissues, is programmed by androgen action earlier in fetal life, before morphological differentiation occurs, and that this might be when TDS disorders originate, especially hypospadias and cryptorchidism. Obviously, this hypothesis cannot be studied directly in humans, and so we tested it using a rat model in which pregnant dams were exposed either to the anti-androgen flutamide or to testosterone, both of which are well documented to affect masculinization in animal studies $(29,32-36)$.

\section{Results}

To investigate the critical window of fetal androgen action in masculinization, rats were exposed to androgens or anti-androgens in specific windows during the last week of gestation (E15.5-E21.5). These encompassed: (a) the period just after onset of testicular testosterone production (E15.5-E17.5, early window [EW]); (b) the period immediately prior to morphological differentiation of reproductive tract tissues (E17.5-E19.5, middle window [MW]); or (c) the period of morphological differentiation of the reproductive tissues in males, defined by the appearance of budding in the prostate (21) and differentiation of the wolffian duct (31) (E19.5-E21.5, late window $[\mathrm{LW}])$. These animals were compared with those exposed to flutamide for the whole period of fetal reproductive development, from onset of androgen production by the testis until birth (E15.5-E21.5, full window [FW]). It is important to note that flutamide is cleared quickly from the body, with a half life of just 5-6 hours in humans (37), and that in rats, flutamide concentrations have significantly decreased 18 hours after exposure (38). We therefore believe that flutamide will no longer be present at effective concentrations in the fetus 24 hours after dosing.

Identification of a programming window for masculinization of the reproductive tract. From the current literature, expectations were that the window of androgen-mediated masculinization would coincide with morphological differentiation of male reproductive tract tissues, which occurs late in pregnancy in the rat ("differentiation window"). We tested this by exposing pregnant rats to the androgen receptor (AR) antagonist flutamide only during this period (E19.5-E21.5, LW). However, this treatment had no effect on the formation of the male reproductive tract tissues, as all reproductive tissues were present in all males (Figure 1). In contrast, we and others have shown that flutamide exposure for the entire period when fetal testicular testosterone is produced (FW) completely blocks reproductive tract masculinization, resulting in the absence of a prostate and seminal vesicles and failure of the phallus to elongate (Figure 1). We now show that this phenotype is a result of impaired androgen action before 
A

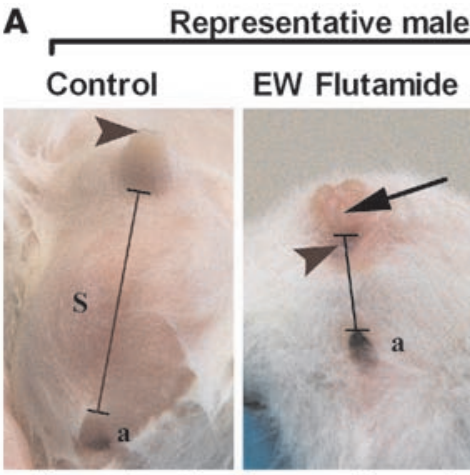

C

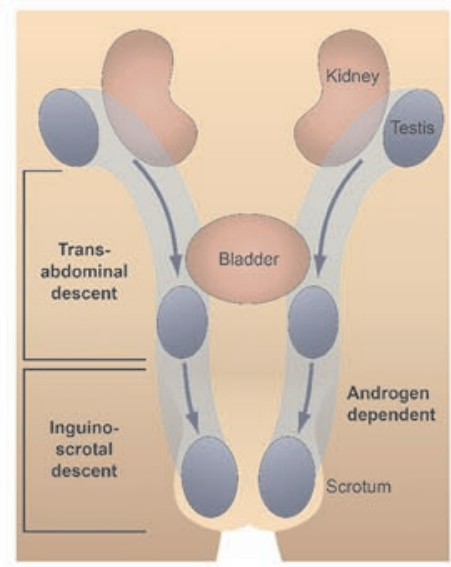

E

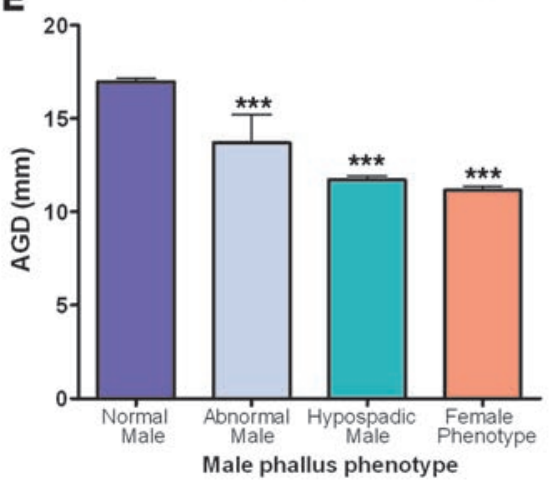

B

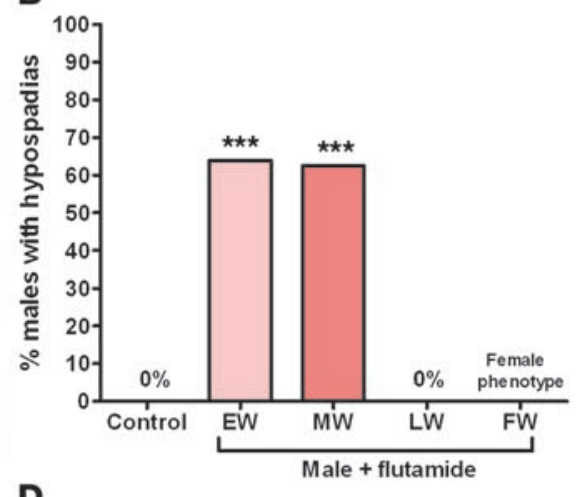

D

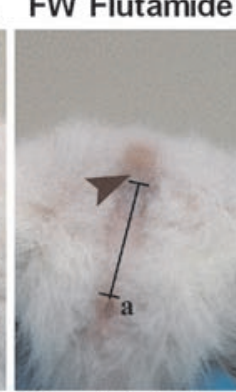

'Female' phallus

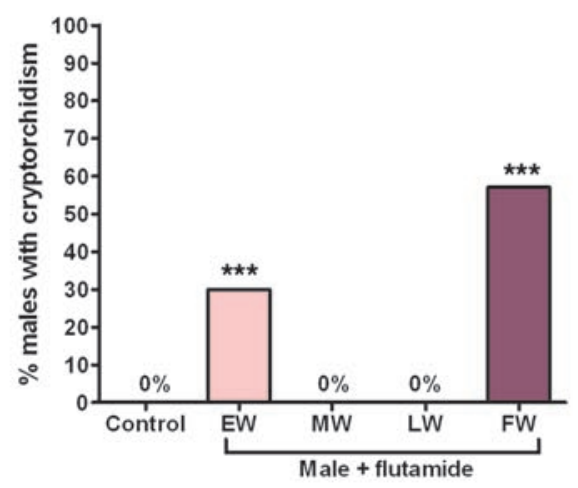

$\mathbf{F}$

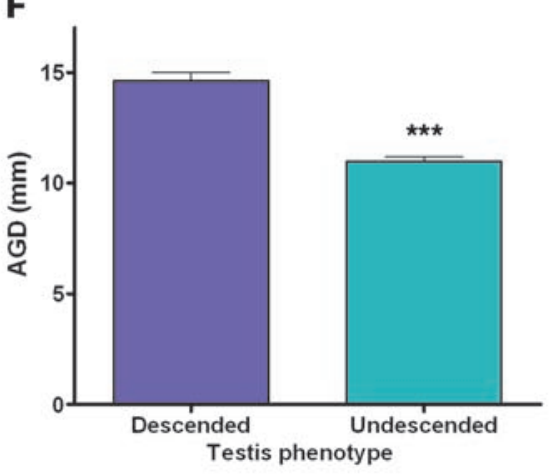

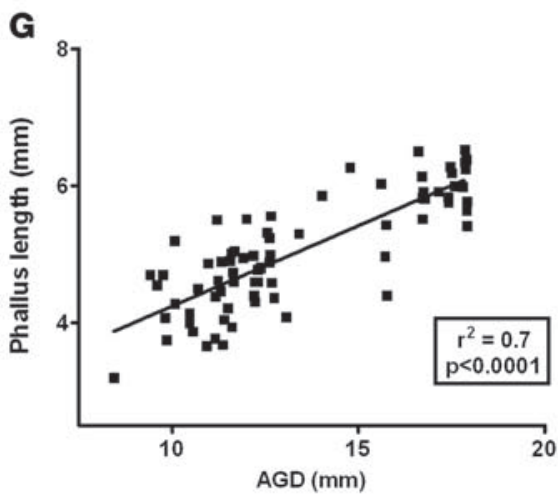

Figure 2

Reproductive congenital abnormalities can only be induced by impaired androgen action in the early programming window. Male rats exposed in utero to flutamide in EW (E15.5-E17.5), MW (E17.5-E19.5), LW (E19.5-E21.5), or FW (E15.5-E21.5) were examined at P25 for hypospadias and cryptorchidism. (A) Images of external genitalia in P25 males demonstrating normal AGD (line) and penis formation, with the urethral opening (arrowhead) at the tip, and scrotal testes (S) in control males compared with reduced AGD, abnormal penis formation with a urethral opening at the base, and undescended testes in EW or FW flutamide-exposed males. Original magnification, $\times 1.4$. (B) Note that EW or MW flutamide induced hypospadias, as evidenced in A by the slit-like opening on the ventral surface of the penis (arrow), while FW-flutamide exposure resulted in a phallus with a female phenotype. (C) Schematic diagram of the phases of normal testis descent. (D) Incidence of cryptorchidism, showing that it can only be induced by flutamide exposure during the EW. Reduced AGD predicted the severity of hypospadias (E) and the incidence of cryptorchidism $(\mathbf{F})$, as well as correlating with reduced penis length $(\mathbf{G})$. Values are mean \pm SEM $(n=8-28$ rats from $2-5$ litters per treatment group). ${ }^{* *} P<0.001$ compared with control male values.

E19.5, as flutamide exposure only in EW or MW impaired reproductive tract masculinization (Figure 1). In these animals, only $7 \%$ and $12 \%$ of males had a ventral prostate and $28 \%$ and $37 \%$ had seminal vesicles, respectively (Figure 1, B and C). Furthermore, these tissues were largely vestigial when present in these animals, and the weight of the ventral prostate and seminal vesicles was dramatically reduced, compared with those from control males $(P<0.001$; Figure 1, D and E). Interestingly, exposure to flutamide in the LW had no effect on ventral prostate weight but significantly reduced seminal vesicle weight $(P<0.001$; Figure 1, D and E); 
A
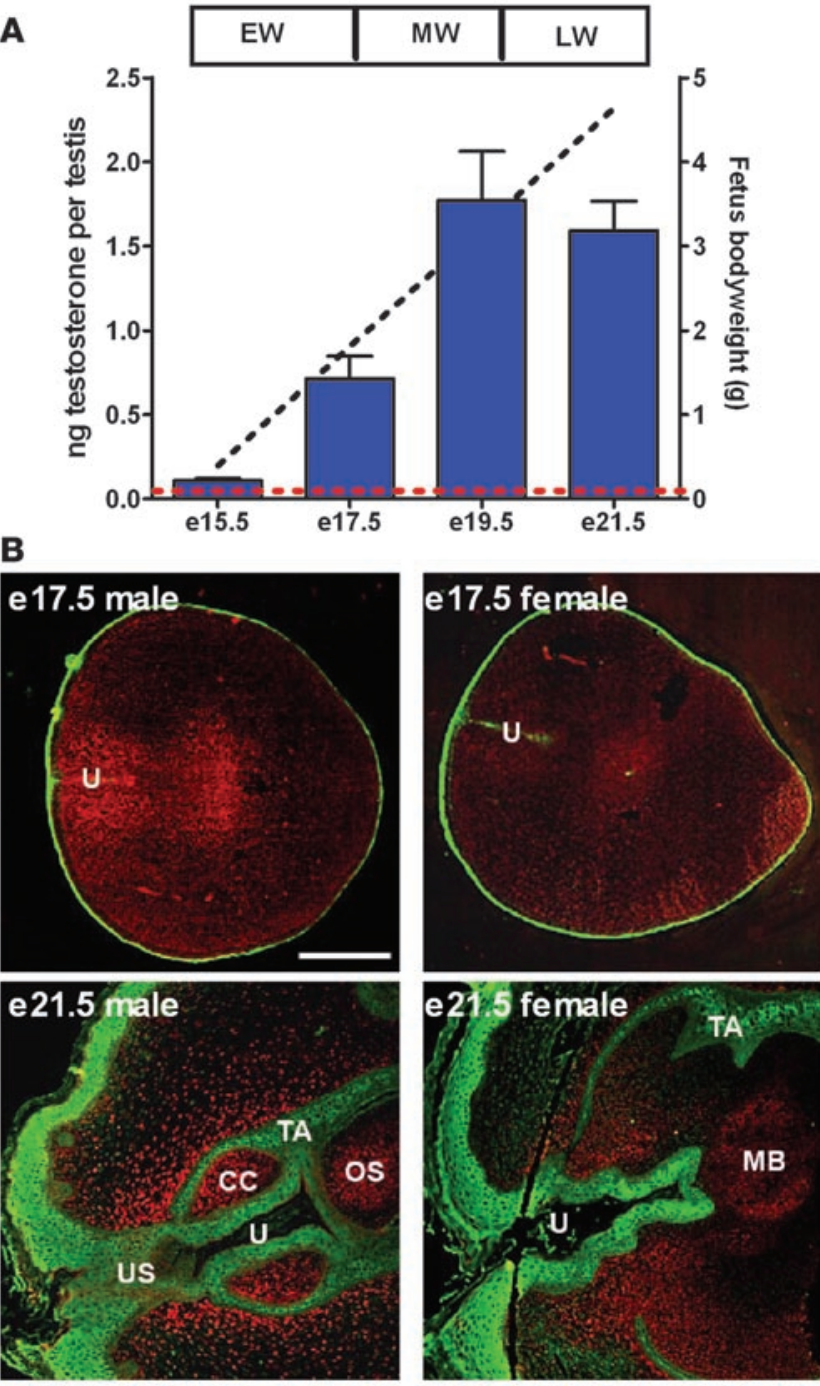

however, this reduction was nowhere near as dramatic as that induced by EW or MW flutamide. Exposure to flutamide in either the EW or MW also significantly reduced the length of the phallus, compared with control males $(P<0.0001$; Figure $1 \mathrm{G})$; this reduction was comparable to that induced by FW flutamide and resulted in a female-length phallus. LW flutamide treatment had no effect on phallus length $(P>0.05$; Figure 1G). Masculinization of anogenital distance (AGD), which is approximately twice as long in males as females in humans (39) and rats (36), was also completely prevented by EW, but not $\mathrm{LW}$, flutamide treatment (Figure 1F); exposure to MW flutamide treatment significantly reduced AGD, compared with control males $(P>0.001$; Figure $1 \mathrm{~F})$, but this reduction was far less dramatic than that induced by $\mathrm{EW}$ treatment. The flutamide-induced reduction in AGD was permanent, as it persisted until adulthood (data not shown). These findings confirmed our hypothesis that masculinization is programmed much earlier than previously assumed, and this occurs before morphological differentiation. Furthermore, this is the first demonstration to our knowledge that this "programming window" is common to all reproductive tract tissues. Our flutamide time window studies show that masculinization of the reproductive tract is programmed in rats during some or all of the

\section{Figure 3}

Androgen concentrations and AR expression in a representative target tissue during the programming and differentiation windows. (A) Fetal testicular testosterone concentration (ng per testis) increases in line with mean fetal body weight (dotted line) between E15.5 and E21.5; red dotted line represents mean ovarian testosterone concentrations from E17.5 females. Note that there was no significant increase in testicular testosterone concentrations at any age compared with values for the previous day. Values are mean $\pm \operatorname{SEM}(n=4-17)$. (B) AR (red) expression in urogenital tubercles in males and females at E17.5 and E21.5, showing that the AR expression pattern is comparable in males and females at both ages. The urogenital tubercle has already begun to differentiate into a penis in the E21.5 male, as evidenced by the presence of the os bone (OS) and the corpus cavernosum (CC). Note also that the urethral folds have begun to fuse in the male to form the urethral seam (US; cytokeratin-stained, green), unlike in the female. $U$, urethra; TA, tunica albuginea; MB, membranous bone. Scale bar: $200 \mu \mathrm{m}$.

period between E15.5 and E19.5. Herein, the term programming window refers to the period prior to E19.5 and encompasses both the early and middle treatment windows.

Relationship of hypospadias and cryptorchidism to the programming window. Since these studies demonstrated that androgen-driven masculinization of all components of the reproductive tract is preprogrammed during a common early programming window, we examined when blockade of fetal androgen action could induce hypospadias or cryptorchidism. We found hypospadias in $64 \%$ and $63 \%$ of males exposed to flutamide treatment in the EW and MW, respectively (Figure 2, A and B). FW flutamide exposure did not induce hypospadias in males but instead induced a completely female phallus phenotype (Figure 2A); this can be considered to be a more severe phenotype than hypospadias. Cryptorchidism was induced in $30 \%$ and $57 \%$ of males exposed to EW and FW flutamide treatment, respectively (Figure 2D). LW flutamide treatment induced neither hypospadias nor cryptorchidism (Figure 2). Importantly, subnormal AGD correlated with incidence and severity of hypospadias $(P<0.001$; Figure $2 \mathrm{E})$, cryptorchidism $(P<0.001$; Figure $2 \mathrm{~F})$, reduced phallus length $\left(r^{2}=0.7\right.$; Figure $\left.2 \mathrm{G}\right)$, and seminal vesicle weight $\left(r^{2}=0.5\right.$; data not shown).

Androgen concentrations and AR expression during the programming and differentiation windows. Our findings show that exposure to flutamide in the LW had little effect on masculinization of the reproductive tract in males. We therefore examined whether the ability of flutamide to block reproductive tract masculinization in the programming but not the differentiation windows could be explained by differences in testicular testosterone production or AR expression. However, testicular testosterone production only increased in parallel with fetal body weight between E15.5 and E21.5 in males (Figure 3A). Moreover, the AR was expressed in reproductive tract tissues from E15.5 to E21.5 in both males and females (Figure 3B and Welsh et al., unpublished observations). Thus, the different reproductive phenotypes induced by flutamide exposure in the programming window (i.e., E15.5-E19.5) compared with the differentiation window (i.e., E19.5-E21.5) cannot be explained by variation in testosterone concentrations or AR expression.

The early programming window also determines experimental masculinization of the reproductive tract in female rats. Since AR is also expressed in reproductive tissues in females and previous studies have shown that inappropriate androgen exposure can masculinize the female fetus $(36,40)$, we investigated whether exposing pregnant rats to exogenous testosterone during the same time windows as 

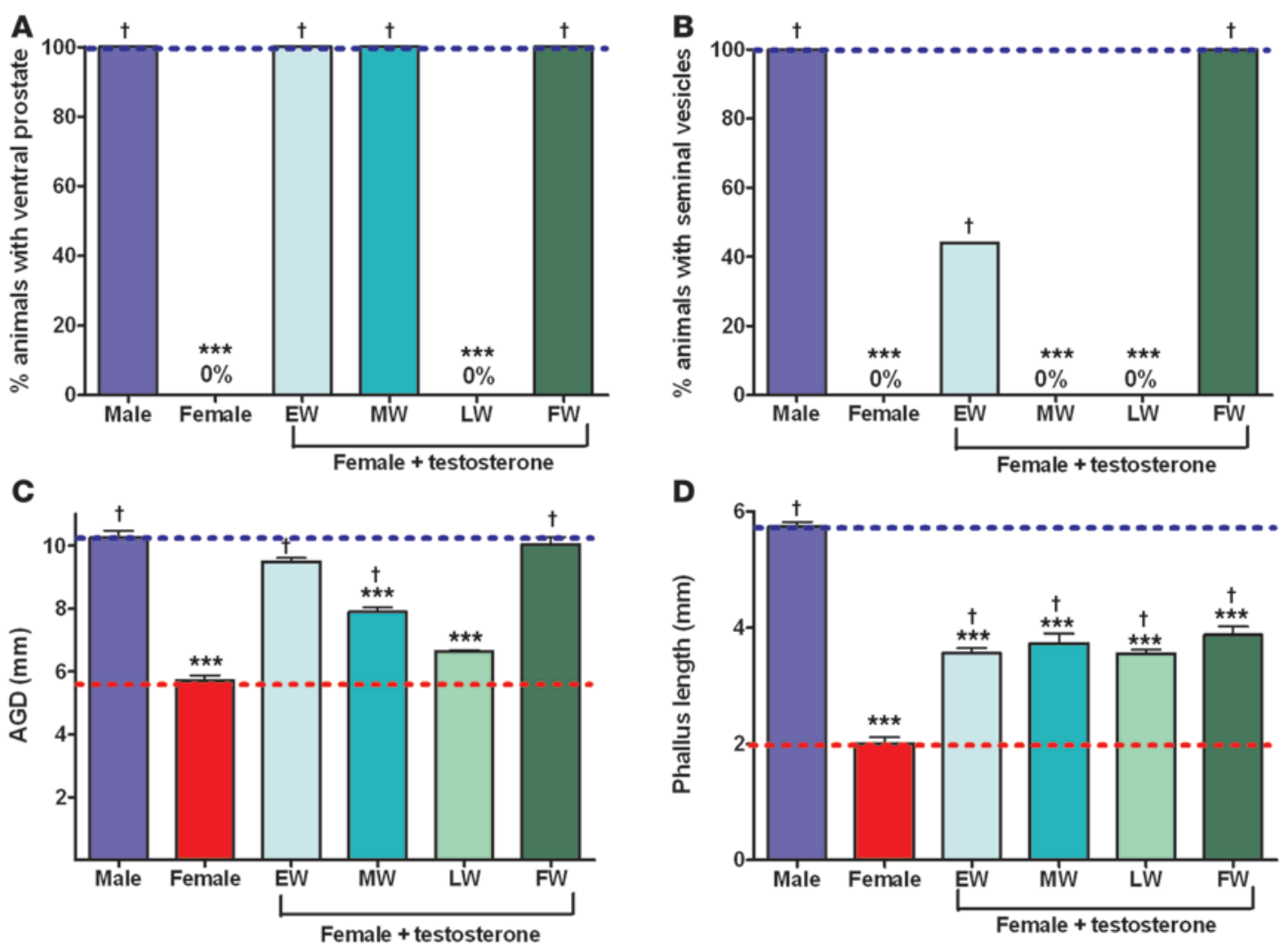

\section{Figure 4}

The early programming window determines experimental masculinization of the reproductive tract in female rats. Reproductive tracts from female rats exposed in utero to testosterone in EW (E15.5-E17.5), MW (E17.5-E19.5), LW (E19.5-E21.5), or FW (E15.5-E21.5) were examined at $\mathrm{P} 17$. Exposure to testosterone during the EW or MW resulted in formation of a ventral prostate (A), while exposure to testosterone during the EW induced seminal vesicle formation (B) and increased AGD in females to a male length (C). Exposure to testosterone during any time window increased phallus length compared with that in control females, but not to a male length (D). Values are mean \pm SEM $(n=8-21$ rats from $2-5$ litters per treatment group). ${ }^{* \star *} P<0.001$ compared with control male values; ${ }^{\dagger} P<0.001$ compared with control female values. Blue dotted line highlights mean control male level; red dotted line highlights mean control female level.

for flutamide could masculinize the reproductive tissues in the female offspring. Females exposed to androgens during the EW (E15.5-E17.5) were completely masculinized, as evidenced by the formation of a prostate and seminal vesicles, increase in AGD to a length normal for males, and increased phallus length. In contrast, exposure to testosterone in the LW (E19.5-E21.5) did not induce prostate or seminal vesicle formation and had no effect on AGD (Figure 4). However, LW testosterone exposure did result in a similar increase in phallus length as did FW testosterone exposure (Figure 4D). Exposure to testosterone in the MW (E17.5-E19.5) induced the formation of a prostate but not seminal vesicles in females, increased AGD but not to a normal male length, and increased phallus length (Figure 4). Therefore, in females, as in males, androgens must act during the programming window (i.e., E15.5-E19.5) to masculinize the prostate, seminal vesicles, and perineum (AGD), but elongation of the phallus is sensitive to androgen action outside of this EW (see below).

Exposure to exogenous testosterone in utero cannot advance or enhance development of reproductive tract tissues in the male. Interestingly, exposure to exogenous testosterone any time during (E15.5E17.5 or E17.5-E19.5) or after (E19.5-E21.5) the programming window did not enhance development of reproductive structures in the male, since AGD, the ventral prostate, and the penis were no larger than in control males (Figure 5). Thus, masculinization in males appears to operate at maximum capacity with normal endogenous testosterone concentrations.

Characterization of the windows of androgen action on phallus development during fetal life. Our flutamide studies showed that the only effect on the male reproductive tract of inhibiting androgen action in the late differentiation window was a $22 \%$ decrease in penis weight compared with that of controls $(P<0.001$; Figure $6 \mathrm{~A})$, while penile length was not affected $(P>0.05$; Figure $1 \mathrm{G})$. Furthermore, histological analysis showed that exposure to flutamide in the EW (E15.5-E17.5), but not the LW (E19.5-E21.5), resulted in a failure of the urethral folds to fuse, resulting in hypospadias (Figure 6C) and a lack of ossification of the penile os bone (Figure $6 \mathrm{C})$. This demonstrates that androgens act during different fetal time windows in males to program penis formation and elongation compared with its subsequent increase in weight.

However in females, phallus (clitoral) weight and length were both sensitive to exogenous androgen action outside of the masculinization programming window, since exposure to testosterone during the EW, MW, or LW resulted in an increase in size comparable to that induced by testosterone exposure for the FW $(P>0.05$; Figure 6B and Figure 4D). Intriguingly, a male-sized phallus at P17 could not be induced by exogenous androgen exposure in females, 
A

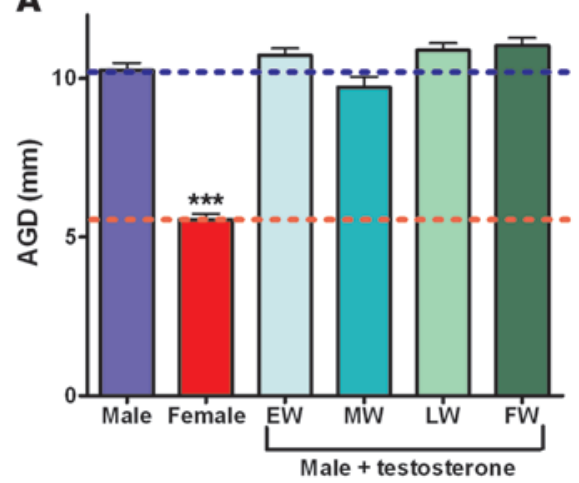

B

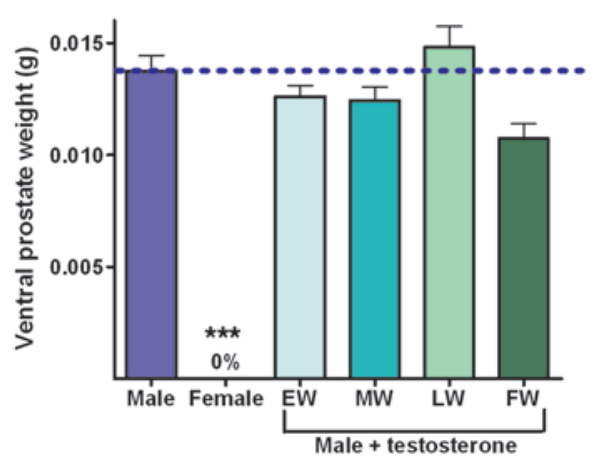

C

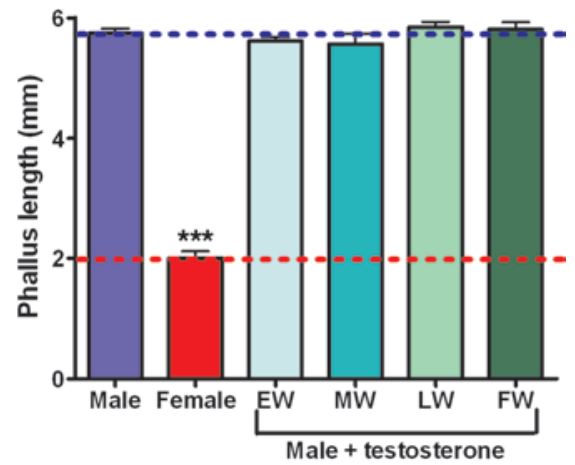

Figure 5

Exposure to exogenous testosterone in utero cannot advance or enhance development of the reproductive tract tissues in the male. Reproductive tracts from male rats exposed in utero to testosterone in EW (E15.5-E17.5), MW (E17.5-E19.5), LW (E19.5-E21.5) or FW (E15.5-21.5), compared with control male and female rats, were examined at P17. AGD (A), ventral prostate weight (B), and phallus length (C) were not increased in males after exposure to testosterone during any time window in fetal life. Values are mean \pm SEM $(n=5-21$ rats from $2-5$ litters per treatment group). ${ }^{* \star \star} P<0.001$ compared with control male values. Blue dotted line highlights mean control male level; red dotted line highlights mean control female level.

regardless of the timing or duration of fetal exposure (Figures 4 and 6); however, our subsequent studies have shown that this is due to a lack of androgen action postnatally (Welsh et al., unpublished observations).

\section{Discussion}

The impetus for the present studies was 2 -fold. First was the need to establish precisely when in fetal life reduced androgen action could induce hypospadias or cryptorchidism, as humans disorders such as these are hypothesized to result from reduced androgen action soon after fetal testis differentiation $(11,14)$. The second stimulus resulted from our unexpected discovery that androgen-driven morphological differentiation of the wolffian duct resulted from androgen action not at the time of differentiation but several days earlier (31); we described this as "programming" by androgens. This prompted us to ask whether similar programming applied to the rest of the reproductive tract (penis, prostate) and led us to hypothesize that androgen-driven masculinization of the various reproductive tract tissues is programmed by androgen action earlier in fetal life, before morphological differentiation of these tissues occurs (Figure 7), and that this is when hypospadias and cryptorchidism originate.

Obviously, this hypothesis could not be tested directly in humans. We therefore tested it using a rat model in which pregnant dams were exposed to either the anti-androgen flutamide or to testosterone; reproductive tract development was then examined in the male and female offspring. These studies largely confirmed our hypothesis that androgen-driven masculinization of all components of the reproductive tract is mediated during a common early programming window (prior to E19.5; Figure 7), as opposed to during the later differentiation window (after E19.5), when morphological differentiation of tissues can be observed. We have also shown for the first time to our knowledge that this masculinization programming window is not restricted to males but also applies to females abnormally exposed to androgens in utero. Furthermore, deficient androgen action only within this early programming window in males can induce the common congenital disorders cryptorchidism and hypospadias. Importantly, we have shown that AGD provides a noninvasive, lifelong "readout" of androgen action specifically within the programming window and predicts incidence of hypospadias and cryptorchidism, as well as penile length and seminal vesicle weight in rats. Cryptorchidism and hypospadias are common in newborn boys, occurring in $2.4 \%-9 \%$ and $0.4 \%-1 \%$ of boys at birth, respectively $(9,10)$; our findings in rats suggest that these disorders are likely to originate early in gestation due to inadequate programming by androgens. Furthermore, our findings strongly encourage measurement of AGD in humans as a readout of androgen action specifically within the masculinization programming window, as AGD may be predictive of adult-onset TDS disorders.

The phenotype we observed in male rats after exposure to flutamide during the FW of fetal reproductive development (i.e., E15.5-E21.5) is comparable to that previously reported by other researchers $(29,41,42)$. We have shown that this phenotype is due to impaired androgen action before E19.5, during which time androgen action is vital to induce the formation and later correct development of all male reproductive tissues in males. To our knowledge, this is the first report of a programming window common to the formation and differentiation of all structures of the male reproductive tract, although there are already published data that agree with this $(43,44)$. However, the earlier data generally refer to only one tissue, and therefore the wider implications that we now illustrate were not fully realized. Our discovery of a common masculinization programming window raises fundamental new questions, such as what regulates this programming window, and can it be affected by factors such as fetal growth restriction (which is a risk factor for both cryptorchidism and hypospadias; refs. 11, 14). Our studies also highlight that there is a fixed time window in which environmental endocrine disruptors could interfere with male reproductive development and identify one key reason why the mechanisms underlying androgen-driven masculinization have previously evaded researchers who, like ourselves, had focused their studies exclusively on the later differentiation window. It is surprising that androgen action during the later differentiation window is unnecessary for normal formation of reproductive tissues in males or for their subsequent differentiation, although a role in determining seminal vesicle and penis size (see below) was identified. 
A
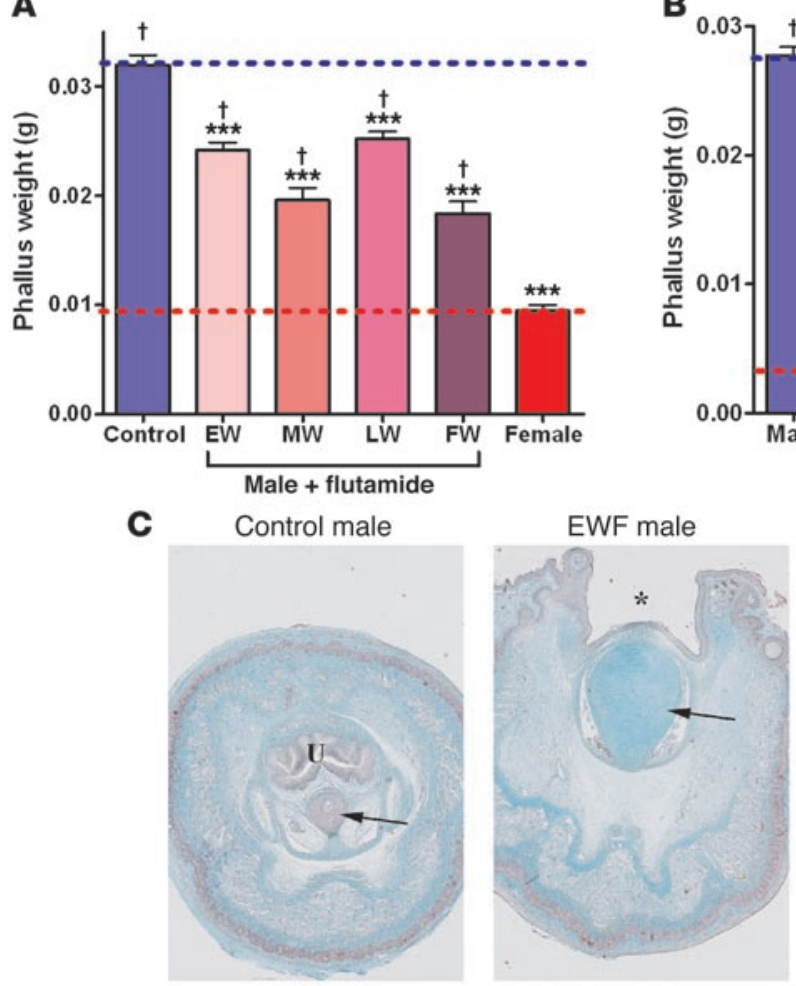

B

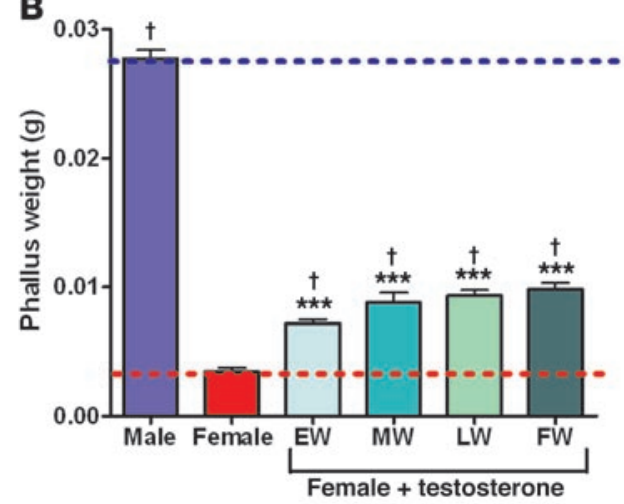

\section{Figure 6}

Characterization of windows of androgen action in phallus development during fetal life. Reproductive tracts were examined at P25 from male rats exposed to flutamide $(\mathbf{A})$ or at P17 from female rats exposed to testosterone (B) in utero during EW (E15.5-E17.5), MW (E17.5-E19.5), LW (E19.5-E21.5), or FW (E15.5-E21.5), compared with control male and female rats. (A) Phallus weight was reduced in male rats by exposure to flutamide during any window between E15.5 and E21.5, compared with that in control males. (B) Testosterone exposure during any window between E15.5 and E21.5 increased phallus weight in female rats, compared with that in control females, but not with male weight. (C) Images of P25 male penis histology showing that EW, but not LW, flutamide affected ossification of the os penis (arrow), indicated by the absence of osteoid cells (stained red by Goldner stain) in the os penis after exposure to EW flutamide. Note also the failure of the urethral folds to fuse in the EW flutamide penis $\left({ }^{*}\right)$, compared with the formation of a patent urethra in the control and LW flutamide male. Original magnification, $\times 20$. Values are mean \pm SEM ( $n=5-28$ rats from $2-5$ litters per treatment group). ${ }^{* * *} P<0.001$ compared with control male values; ${ }^{\dagger} P<0.001$ compared with control female values. Blue dotted line highlights mean male level; red dotted line highlights mean female level.

Since our findings showed that LW flutamide exposure had little effect on masculinization of the reproductive tract, we investigated whether the ability of flutamide to block masculinization during the programming but not the differentiation window could be explained by differences in the concentration of endogenous testosterone available to compete with flutamide for AR binding or differences in levels of AR expressed in the tissues. However, testicular testosterone concentrations simply increased in parallel with fetal body weight between E15.5 and E21.5, and it has been shown previously that fetal serum testosterone concentrations are relatively stable between E17.5 and E21.5 in rats (18). Moreover, the AR was expressed in all reproductive tract tissues in both males and females at all ages studied, a pattern consistent with published data $(31,45,46)$. Furthermore, the dose of flutamide used in these studies was 4- to 15 -fold greater than that required to abolish prostate development (29) or to prevent masculinization of AGD in rats (ref. 41 and our unpublished observations). Therefore, the reproductive phenotype observed after exposure to flutamide in

the EW compared with that in males exposed to flutamide in the LW cannot be due to differences in the effectiveness of flutamide to antagonize androgen action, but rather must be due to different roles for androgen in each of the fetal time windows. This conclusion is further substantiated by our findings regarding the restricted time window in which exogenous testosterone can masculinize females.

It is well established that inappropriately high androgen exposure can masculinize the female fetus $(36,40)$. A new clinically relevant question that emerged from the present studies in males was whether experimental masculinization of the reproductive tract in the female fetus is confined to the same early programming window as in males. We investigated this by exposing pregnant rats to exogenous testosterone and examining the impact on reproductive development in the female offspring. Females exposed to testosterone during the masculinization programming window (i.e., E15.5-E19.5) developed prostates and seminal vesicles and had an increased AGD and increased phallus length. In contrast, testosterone exposure in the later male differentiation window (i.e., E19.5-E21.5) did not masculinize the female reproductive tract, although it did increase the size of the phallus, as discussed below. Therefore, in females, as in males, androgens must act within the programming window if they are to masculinize the reproductive tract. The factor(s) determining the masculinization programming window is unknown, but it is likely to involve regulation of the onset of AR expression, is not sexually dimorphic, and is a potential target for disruption of masculinization. Interestingly, exposure to exogenous testosterone any time between E15.5 and E21.5 did not enhance development of reproductive structures in the male; for example, penis size was no larger than that in control males. This demonstrates that reproductive tract masculinization in normal males involves maximal AR activation.

Our studies also provide new insight into phallus development. We have shown for the first time to our knowledge that phallus length and weight appear to be differentially regulated, since androgen action in the fetal differentiation window (i.e., E19.5E21.5) affected phallus weight, but not length, in males. Furthermore, histological analysis demonstrated that ossification of the penile os bone in males was prevented by exposure to flutamide in the early or middle treatment windows, but not in the LW. This 


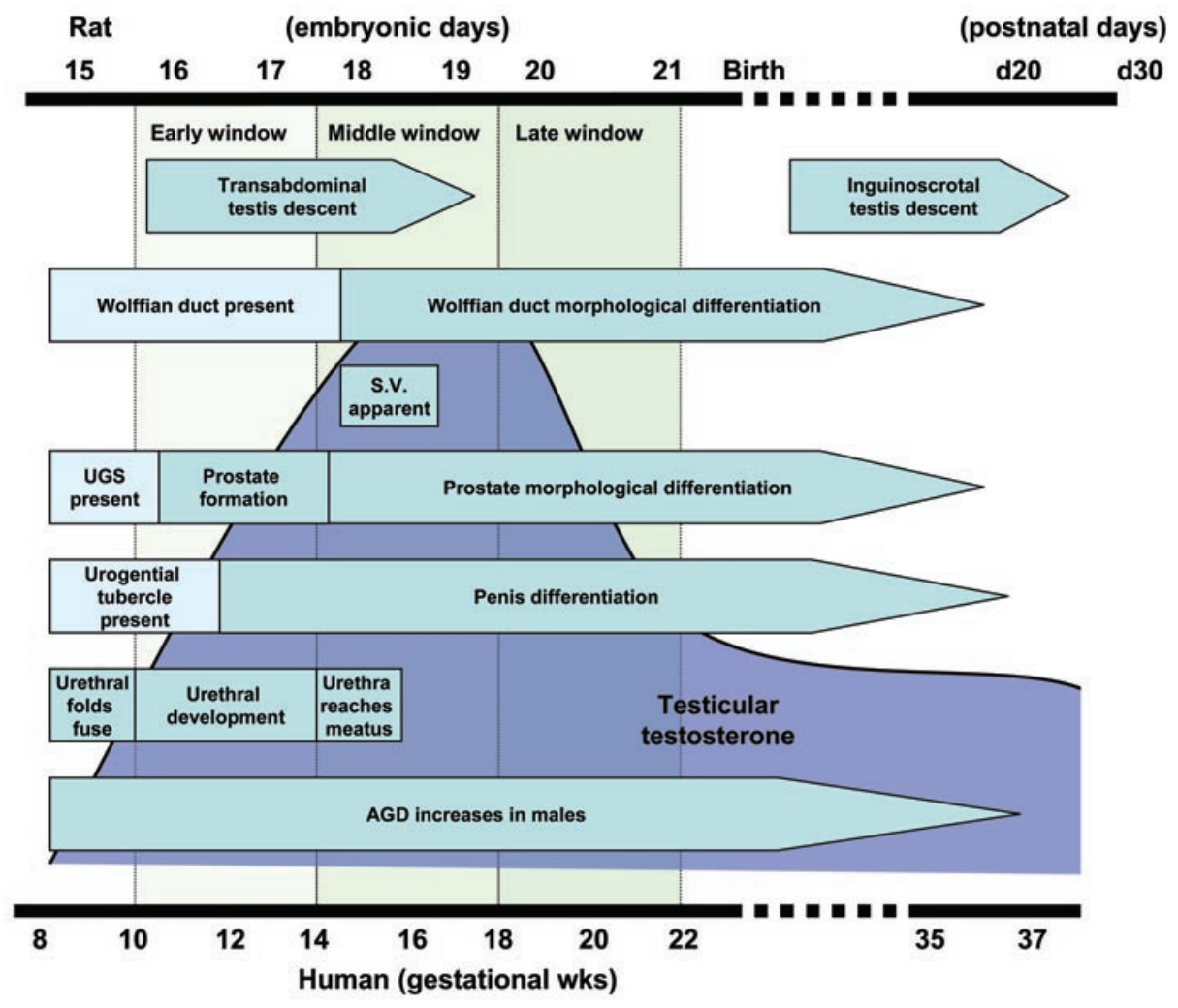

Figure 7

Schematic diagram illustrating the timing of male reproductive tract development in humans and rats in relation to testicular testosterone production/levels; timings are based on data in the literature (17-23, $25,26,31)$. Note that wolffian ducts, urogenital sinus (UGS), and urogenital tubercle are present initially in both sexes. The time windows used for the present rat treatments are also shown. The masculinization programming window in rats occurs between E15.5 and E19.5. S.V., seminal vesicles. agrees with data from the Tfm mouse showing that ossification of the penile os is an androgen-mediated event (47). It is important to note that the human penis has no os bone, but it is likely that histological differentiation of the human penis will also be mediated by androgen action early in fetal life. Importantly, our results show that blockade of androgen action only in the masculinization programming window can induce hypospadias, while blockade in the later differentiation window had no discernible effect on gross morphology of the penis. Together, these data demonstrate that androgen action before E19.5 is vital for programming penis formation and elongation in rats, whereas androgen action throughout fetal life (and some of postnatal life) can mediate the subsequent increase in penile weight. This agrees with evidence that penile development in humans correlates with testosterone concentrations neonatally (48). The situation in the female, however, appears to be slightly different from that in males, in that female phallus (clitoral) weight and length both remained sensitive to exogenous androgen action after the masculinization programming window. This is consistent with monkey and human data showing that clitoromegaly can be induced by androgens in late fetal life $(6,27,40)$. This could mean that there are fundamental differences in the responsiveness of the undifferentiated male and female phallus, and this is currently under investigation. In the present studies, fetal androgen exposure in females was unable to induce a male-sized phallus when measured at P17, regardless of the timing or duration of exposure. However, our subsequent studies have shown that this is due to a lack of androgen action postnatally, as occurs in normal males (data not shown). Although our data show unequivocally that formation of a normal penis is critically dependent on androgen action during the early masculinization programming window, they also show that late fetal and/or postnatal androgen action is required in order for the phallus to reach its full male size. This agrees with previously published studies in humans (48).

Interestingly, exposure to flutamide during the FW of fetal reproductive development (E15.5-E21.5) resulted in formation of a phallus in males that appeared grossly female, with no sign of hypospadias. In contrast, exposure to flutamide only in the EW (E15.5-E17.5) or MW (E17.5-E19.5) resulted most commonly in hypospadias, though some animals showed a female phenotype. This suggests that the critical window for androgen action in penis formation is not restricted just to the EW (E15.5-E17.5), but that androgen action for at least some of the MW (E17.5-E19.5) is also essential. This gradient of phallus development has also been reported in humans with androgen insensitivity syndrome, in which structures range from a normal male phallus to a hypospadiac male phallus to an apparently female phallus, depending on the degree of androgen action (49). However, the fundamental finding from the present studies is that androgen action before E19.5 in rats is vital to induce penis formation and that androgen action after this age cannot result in normal penis formation, even though it can modify phallus size.

Perhaps our most unexpected finding was that transinguinal testicular descent was also programmed by androgen action in the early programming window. This was surprising, as this androgen-dependent phase of testis descent is a late event in the rat, occurring at postnatal week 3 (26), almost a month after the masculinization programming window (Figure 7). Nevertheless, our findings agree with similar previous observations (44), and additional studies have also shown that postnatal blockade of androgen action during the period of actual transinguinal testis descent does not induce cryptorchidism (22). Transinguinal testis descent 


\section{Table 1}

Explanations of classification of testis descent and penis morphology

\begin{tabular}{|c|c|c|}
\hline Parameter & Classification & Description \\
\hline \multirow[t]{2}{*}{ Testis position } & Cryptorchid & $\begin{array}{l}\text { Testes located in the abdomen, inguinal (beside the bladder), or ectopic outside the } \\
\text { body cavity (e.g., in the femoral canal) }\end{array}$ \\
\hline & Scrotal & $\begin{array}{l}\text { Testes located in the scrotum or found in the inguinal canal but could be pushed into } \\
\text { the scrotum }\end{array}$ \\
\hline \multirow[t]{4}{*}{ Phallus external morphology } & Normal male & $\begin{array}{l}\text { Urethral opening at the tip of the phallus; phallus long and cylindrical; obvious internal } \\
90^{\circ} \text { S-shaped bend in the phallus }\end{array}$ \\
\hline & Abnormal male & $\begin{array}{l}\text { Urethral opening at the tip of the phallus; phallus long and cylindrical; internal } 90^{\circ} \\
\text { S-shaped bend not apparent in the phallus; phallus fused internally on the ventral } \\
\text { surface to the body wall }\end{array}$ \\
\hline & Hypospadiac male & $\begin{array}{l}\text { Urethral opening on the ventral surface of the phallus or perineum; phallus long and } \\
\text { cylindrical; internal } 90^{\circ} \mathrm{S} \text {-shaped bend not apparent in the phallus }\end{array}$ \\
\hline & Female phenotype & $\begin{array}{l}\text { Female urethral opening; phallus flat and wide; internal } 90^{\circ} \text { S-shaped bend not apparent } \\
\text { in the phallus }\end{array}$ \\
\hline
\end{tabular}

in humans also occurs considerably later (gestation weeks 27-35; refs. 24,25 ) than reproductive tract differentiation (Figure 7 ), and it is this final phase of testis descent that is androgen dependent and is most commonly affected in cryptorchid boys (26). If, as in the rat, transinguinal testis descent in humans results from androgen action during the masculinization programming window, then our results suggest that incomplete or delayed transinguinal testis descent, which is common $(2.4 \%-9 \%)$ in boys at birth (10), reflects impaired androgen action much earlier in pregnancy than was previously suspected. This possibility could be addressed by investigation of the relationship between incomplete transinguinal testis descent at birth and AGD, as discussed below. One puzzling observation from our studies is that flutamide exposure during any of our time windows only induced cryptorchidism (which was frequently unilateral) in 35\%-60\% of males, consistent with incidence rates in previous studies (26). This may mean that there is not an absolute requirement for androgen action in transinguinal testis descent or that other unknown factors may be able to compensate for deficient androgen action in the process. This merits further investigation.

Many previous animal studies have used AGD as an index of altered fetal androgen action (30, 31, 41-43). However, we have shown that AGD can only be affected in rats by manipulation of androgen action between E15.5 and E19.5, since treatment between E19.5 and E21.5 had no effect. AGD can therefore provide new insights into androgen action during this "hidden" period of life and thus will be useful clinically as a noninvasive measure of androgen action specifically within the masculinization programming window, but not during later fetal or neonatal life. For example, AGD may not be a reliable guide in decision making about sex of rearing in cases of ambiguous genitalia at birth, as androgeninduced brain masculinization is a late fetal event in humans and monkeys $(50,51)$ and occurs late in fetal and early postnatal life in rats (52); it is therefore likely to be independent of the programming that we describe and so may not correlate with AGD. We have shown, however, that subnormal AGD correlates with incidence and severity of hypospadias, cryptorchidism, and reduced phallus length and seminal vesicle weight in males exposed in utero to flutamide. Furthermore, in a rat model of TDS (12) based on in utero exposure to di(n-butyl) phthalate, we found a relationship between reduced AGD and occurrence of hypospadias and cryptorchidism similar to that revealed in the present studies; moreover, in this model, AGD also correlates with adult rat testis size and therefore sperm production (Sharpe et al., unpublished observations). AGD at birth could therefore be useful to indicate abnormal androgen action during the critical masculinization programming window. Furthermore, if it is accurate to link the origins of TDS to low fetal androgen actions (11), reduced AGD might also predict the risk of adult-onset disorders such as low sperm counts or testicular germ cell cancer. Since we, and others $(41,53)$, have shown that fetally induced changes in AGD are permanent, AGD could be measured in adulthood as a reflection of androgen action during the masculinization programming window. In this regard, Swan et al. (54) have shown that AGD in newborn boys correlates with gestational exposure to phthalates, which, in rat studies, have been shown to reduce fetal testosterone production and AGD (12, 55-58). Moreover, a recent study (59) has shown that AGD is reduced in boys with hypospadias or cryptorchidism. Together, these findings provide strong incentive for clinical investigations of altered AGD in patients with postulated TDS disorders, such as young men with testicular germ cell cancer; confirmation that AGD was subnormal in such men would provide the grounds for identifying those at future risk of this cancer by measurement of AGD neonatally, a noninvasive and practically easy screen.

In conclusion, our studies identify a common early programming window in rats in which androgen action must occur to masculinize all components of the reproductive tract and enable their correct later development in males. This masculinization programming window is not restricted to males but also applies to females abnormally exposed to androgens in utero. Furthermore, deficient androgen action only within this early programming window can induce the common congenital disorders cryptorchidism and hypospadias. The occurrence of such a programming window in humans remains to be proven, but based on parallels with the rat, it is likely to be between 8 and 14 weeks' gestation (Figure 7). Importantly, we have shown that AGD provides a noninvasive, lifelong readout of androgen action only within the masculinization programming window and predicts incidence of hypospadias and cryptorchidism, as well as penile length and seminal vesicle weight in rats. AGD should therefore be clinically useful in assessing androgen exposure early in gestation, as an indicator of reproductive tract masculinization and its congenital 
disorders and as a potential predictor of adult-onset reproductive disorders. Our detailed studies on androgen action during different developmental stages have also revealed one reason why the cellular mechanisms underlying androgen-driven masculinization have previously evaded researchers who focused exclusively on the later differentiation window. They also identify the time period when environmental endocrine disruptors could interfere with male reproductive development and support the hypothesis that TDS disorders that manifest at birth arise early in gestation in association with subnormal androgen action.

\section{Methods}

Rats. Wistar rats were bred and maintained in our own animal house under standard conditions of care and use under licensed approval from the UK Home Office. Animals had access ad libitum to water and a soy-free breeding diet (SDS). Timed matings were established, and the presence of a vaginal plug was defined as E0.5. Pregnant dams were either untreated and fetuses recovered for testosterone assays and AR analysis or treated (as detailed below), and offspring were examined postnatally.

In vivo studies. A total of 39 pregnant dams were randomly allocated to treatment groups and dosed daily between 08:30 and 10:00 according to maternal body weight with (a) flutamide (Sigma-Aldrich) at $100 \mathrm{mg} / \mathrm{kg}$ by oral gavage in $1 \mathrm{ml} / \mathrm{kg}$ corn oil $/ 2.5 \%$ DMSO ( $n=15$ litters); (b) testosterone propionate (Sigma-Aldrich) at $20 \mathrm{mg} / \mathrm{kg}$ by subcutaneous injection in $0.4 \mathrm{ml} / \mathrm{kg}$ corn oil $(n=14)$; or (c) vehicle control ( $n=10$ litters). This dose of flutamide severely impairs male reproductive tract development $(29,31)$ and is 4 - to 15 -fold greater than that required to abolish prostate development (29) or to prevent masculinization of AGD in rats (ref. 41 and our unpublished observations). The dose of testosterone propionate (TP) used was based on previous results showing that in utero exposure to doses above 3-4 mg/kg completely masculinized AGD in female rats (ref. 36 and our unpublished observations). The dose of testosterone used in our studies frequently induces dystocia, so to avoid this problem, fetuses were caesarean-derived and cross-fostered to untreated/control mothers that had delivered within the last 6 hours.

A pilot study was undertaken to compare FW, EW, and LW flutamide treatment (see below) and to sample offspring at various postnatal ages to identify optimal time points for subsequent analysis as well as determining the persistence of reproductive tract abnormalities through to adulthood. A total of 8 dams were exposed to flutamide in either the FW ( $n=3$ litters), EW ( $n=2$ litters), or LW ( $n=3$ litters); offspring were examined for the presence of male reproductive tissues and incidence of hypospadias and cryptorchidism and AGD was measured. These studies demonstrated that all abnormalities could be identified at P25 and persisted until adulthood. The data from this pilot study are not reported in this article but gave comparable results to those presented herein.

For the present studies, dosing was undertaken in specific time windows in the last week of gestation (E15.5-E21.5) that encompassed either (a) the period just after onset of testicular testosterone production (E15.5-E17.5, EW); (b) the period immediately prior to morphological differentiation of reproductive tract tissues (E17.5-E19.5, MW); or (c) the period of morphological differentiation of the reproductive tissues in males, defined by the appearance of budding in the prostate (21) and differentiation of the wolffian duct (31) (E19.5-E21.5, LW). Data were compared with those from animals exposed to flutamide for the whole period from onset of androgen production by the testis until birth (E15.5-E21.5, FW). Dams were checked daily for signs of toxicity and their body weights recorded daily throughout the dosing regime. Male and female offspring were subsequently evaluated postnatally: testosterone-exposed rats were examined at $\mathrm{P} 17$, as this is prior to the onset of puberty in rats and therefore minimizes any confounding effects of endogenous pubertal hormones (in females), while flutamideexposed rats were not examined until P25, since testis descent into the scrotum occurs at P20-P23 in rats (26).

Dissection of fetal tissues. Dams $(n=22)$ were killed by inhalation of carbon dioxide and subsequent cervical dislocation. Fetuses were recovered, decapitated, and placed in ice-cold PBS (Sigma-Aldrich) prior to recovery of gonads, urogenital tubercles, urogenital sinuses, and wolffian and/or müllerian ducts from males and females. Gonads were snap-frozen for subsequent testosterone assay (see below), while all other tissues were fixed in Bouin's fixative for 1 hour, then transferred into $70 \%$ ethanol and processed for 17.5 hours in an automated Leica TP1050 processor and embedded in paraffin wax.

Dissection of postnatal tissues. Male and female offspring were killed at P17 ( $n=125$ rats) or $\mathrm{P} 25$ ( $n=75$ rats) by inhalation of carbon dioxide and subsequent cervical dislocation. All endpoints were examined in all rats. No significant difference was noted in offspring body weight between treatment groups; therefore, no correction factor was necessary for any parameter. Rats were sexed by the presence or absence of testes, as testis formation is not dependent on androgen action and so would not be affected by the treatments used in this study. Rats were weighed, and AGD was measured using digital calipers (Faithfull Tools), as it is widely believed that AGD reflects the degree of masculinization of the animal $(41,43)$. Rats were examined for testis position and penis morphology (Table 1), prior to recovery of reproductive tissues.

The phallus was examined for abnormalities prior to the prepuce and skin being removed and the phallus being recovered; it was consistently severed at the bend where the urethra enters the body. Phallus length was measured using digital calipers and then weighed prior to fixation in Bouin's fixative for 2 hours and processed as above. To recover postnatal reproductive tracts, the abdomen of the supine male rat was opened, and testes, seminal vesicles, ventral prostate, and adrenals were examined for gross morphology, then removed from the animal. Tissue wet weight was recorded prior to fixation in Bouin's fixative for 2 hours and tissue processed as above. Epididymides and vas deferens were examined for gross morphology and showed abnormalities in flutamide-exposed animals comparable to those previously reported $(29,31,42)$; for the present studies, seminal vesicles were weighed and analyzed as representative wolffian duct-derived differentiated tissues.

Goldner. Standard Goldner histological staining was performed on paraffin-embedded phalluses from control and flutamide-exposed male rats (P25) in order to establish normality of penile differentiation, for example, ossification of the penile os bone. Sections were deparaffinized and rehydrated as previously detailed (31) prior to staining with Weigert hematoxylin (blue nuclei), ponceau-fuchsin-azophloxin (red osteoids), and light green solution (green mineralized bone), according to the standard Goldner method. Representative phalluses from at least 3 animals from at least 2 litters were examined, and sections were processed in parallel on at least two occasions.

Testicular testosterone assay. The tiny volume of blood in fetal rats, especially at E15.5-E18.5, makes it difficult to measure serum testosterone concentrations (18). Testicular testosterone levels were therefore measured by RIA, as described previously (12). Testes are too small at E15.5 ( $n=4$ litters) and E17.5 ( $n=5$ litters) to accurately measure testosterone concentrations in individual testes; therefore, one testis from each fetus was pooled within each litter. Testosterone concentrations were measured in individual testes at E19.5 ( $n=6$ rats from 3 litters) and E21.5 ( $n=17$ rats from 4 litters).

Double fluorescence immunohistochemistry for cytokeratin and AR. Bouin'sfixed reproductive tissues recovered from fetuses from untreated or vehicle control dams at E17.5 (during the programming window; $n=3$ litters) and E21.5 (during the differentiation window; $n=3$ litters) were embed- 
ded in paraffin wax, sectioned $(5 \mu \mathrm{m})$, and floated onto slides coated with 2\% 3-aminopropyltriethoxysilane (Sigma-Aldrich), prior to performing double immunohistochemistry for cytokeratin and AR. Sections were deparaffinized and rehydrated and antigen retrieval performed as previously detailed (31). Nonspecific binding sites were blocked by incubating sections in normal goat serum (NGS; Autogen Bioclear UK Ltd.) diluted 1:4 in PBS containing 5\% BSA (Sigma-Aldrich) for 1 hour, then incubated overnight at $4{ }^{\circ} \mathrm{C}$ with anti-pan-cytokeratin antibody (Sigma-Aldrich) diluted 1:200 and anti-AR antibody (Santa Cruz Biotechnology Inc.) diluted 1:50 in NGS/PBS/BSA. AR immunostaining was detected with biotinylated goat anti-rabbit IgG secondary antibody (DAKO) diluted 1:500 in NGS/PBS/BSA, followed by streptavidin-conjugated Alexa Fluor 546, also diluted 1:200 in PBS, to produce red fluorescence. Cytokeratin immunoexpression was detected using goat anti-mouse secondary antibody directly conjugated with Alexa Fluor 488 diluted 1:200 in PBS, to produce green fluorescence. Sections were mounted in Mowiol mounting medium (Calbiochem). Fluorescent images were captured using a Zeiss LSM 510 Meta Axiovert 100M confocal microscope (Carl Zeiss Ltd.). To ensure reproducibility of results and allow accurate comparison of immunostaining, representative reproductive tissues from at least 3 animals from 2 litters were used, and sections from male and female fetuses were processed in parallel on at least 2 occasions. Appropriate negative controls were included, whereby the primary antibody was replaced by normal goat serum alone, to ensure that any staining observed was specific; none of the antibodies used showed other than minor nonspecific staining. For AR expression, the genital tubercle/phallus was used as a rep- resentative reproductive tract tissue, but similar results were also obtained for wolffian duct-derived tissues (data not shown).

Statistics. Data were analyzed using GraphPad Prism version 5 (Graph Pad Software Inc.). Values are expressed as mean \pm SEM. Normality was confirmed using KS normality test, then data were analyzed using a 1-tailed unpaired $t$ test or 1-way ANOVA, followed by Bonferroni posthoc tests. Correlation between AGD and penile length was analyzed using linear regression modeling. In situations in which significant heterogeneity of variance was detected, data were normalized by log transformation, prior to statistical analysis. A $P$ value of less than 0.05 was considered significant.

\section{Acknowledgments}

We thank Ian Swanston for performing the testosterone radioimmunoassay and Sheila McPherson for technical assistance optimizing the Goldner staining protocol. We thank the Medical Research Council UK for funding these studies.

Received for publication October 16, 2007, and accepted in revised form January 23, 2008.

Address correspondence to: Michelle Welsh, MRC Human Reproductive Sciences Unit, Centre for Reproductive Biology, The Queen's Medical Research Institute, 47 Little France Crescent, Edinburgh EH16-4TJ, United Kingdom. Phone: 44-131-242-9125; Fax: 44-131-242-6231; E-mail: m.welsh@hrsu.mrc.ac.uk.
1. Koopman, P., Gubbay, J., Vivian, N., Goodfellow, P., and Lovell-Badge, R. 1991. Male development of chromosomally female mice transgenic for Sry. Nature. 351:117-121.

2. Lovell-Badge, R., and Robertson, E. 1990. XY female mice resulting from a heritable mutation in the primary testis-determining gene, Tdy. Development 109:635-646.

3. Capel, B. 2000. The battle of the sexes. Mech. Dev. 92:89-103.

4. Jost, A. 1972. A new look at the mechanisms controlling sex differentiation in mammals. Johns Hopkins Med. J. 130:38-53.

5. Hughes, I.A., and Deeb, A. 2006. Androgen resistance. Best Pract. Res. Clin. Endocrinol. Metab. 20:577-598.

6. Sarafoglou, K., and Ostrer, H. 2000. Clinical review 111: familial sex reversal: a review. J. Clin. Endocrinol. Metab. 85:483-493.

7. Virtanen, H.E., et al. 2007. Development and descent of the testis in relation to cryptorchidism. Acta Paediatr. 96:622-627.

8. Baskin, L.S., Himes, K., and Colborn, T. 2001. Hypospadias and endocrine disruption: is there a connection? Environ. Health Perspect. 109:1175-1183.

9. Boisen, K.A., et al. 2005. Hypospadias in a cohort of 1072 Danish newborn boys: prevalence and relationship to placental weight, anthropometrical measurements at birth, and reproductive hormone levels at three months of age. J. Clin. Endocrinol. Metab. 90:4041-4046.

10. Boisen, K.A., et al. 2004. Difference in prevalence of congenital cryptorchidism in infants between two Nordic countries. Lancet. 363:1264-1269.

11. Skakkebaek, N.E., Rajpert-De Meyts, E., and Main, K.M. 2001. Testicular dysgenesis syndrome: an increasingly common developmental disorder with environmental aspects. Hum. Reprod. 16:972-978.

12. Fisher, J.S., Macpherson, S., Marchetti, N., and Sharpe, R.M. 2003. Human 'testicular dysgenesis syndrome': a possible model using in-utero exposure of the rat to dibutyl phthalate. Hum. Reprod. 18:1383-1394.

13. Sharpe, R.M., and Skakkebaek, N.E. 2003. Male reproductive disorders and the role of endocrine disruption: advances in understanding and identification of areas for future research. Pure Appl. Chem. 75:2023-2038.

14. Sharpe, R.M. and Skakkebaek, N.E. 2008. Testicular dysgenesis syndrome: mechanistic insights and potential new downstream effects. Fertil. Steril. In press.

15. Peets, E.A., Henson, M.F., and Neri, R. 1974. On the mechanism of the anti-androgenic action of flutamide (alpha-alpha-alpha-trifluoro-2-methyl4'-nitro-m-propionotoluidide) in the rat. Endocrinology. 94:532-540.

16. Schaufele, F., et al. 2005. The structural basis of androgen receptor activation: intramolecular and intermolecular amino-carboxy interactions. Proc. Natl. Acad. Sci. U. S. A. 102:9802-9807.

17. Siiteri, P.K., and Wilson, J.D. 1974. Testosterone formation and metabolism during male sexual differentiation in the human embryo. J. Clin. Endocrinol. Metab. 38:113-125.

18. Habert, R., and Picon, R. 1984. Testosterone, dihydrotestosterone and estradiol-17 beta levels in maternal and fetal plasma and in fetal testes in the rat. J. Steroid Biochem. 21:193-198.

19. George, F.W., and Wilson, J. 1994. Gonads and ducts in mammals. In The physiology of reproduction. E. Knobil and J.D. Neill, editors. 2nd ed. Raven Press. New York, New York, USA. 3-28.

20. Reyes, F.I., Winter, J.S., and Faiman, C. 1973. Studies on human sexual development. I. Fetal gonadal and adrenal sex steroids. J. Clin. Endocrinol. Metab. 37:74-78.

21. Price, D. 1936. Normal development of the prostate and seminal vesicles of the rat, with a study of experimental post-natal modifications. Am.J. Anat. 60:79-125.

22. Inomata, T., Eguchi, Y., and Nakamura, T. 1985. Development of the external genitalia in rat fetuses. Jikken Dobutsu. 34:439-444.

23. Jirasek, J.E. 1977. Morphogenesis of the genital system in the human. Birth Defects Orig. Artic. Ser. 13:13-39.
24. Wyndham, N.R. 1943. A morphological study of testicular descent. J. Anat. 77:179-188.

25. Wilhelm, D., and Koopman, P. 2006. The makings of maleness: towards an integrated view of male sexual development. Nat. Rev. Genet. 7:620-631.

26. Amann, R.P., and Veeramachaneni, D.N. 2007. Cryptorchidism in common eutherian mammals. Reproduction. 133:541-561.

27. Goy, R.W., Bercovitch, F.B., and McBrair, M.C. 1988. Behavioral masculinization is independent of genital masculinization in prenatally androgenized female rhesus macaques. Horm. Behav. 22:552-571.

28. Welsh, M., Saunders, P.T., Marchetti, N.I. and Sharpe, R.M. 2006. Androgen-dependent mechanisms of Wolffian duct development and their perturbation by flutamide. Endocrinology. 147:4820-4830.

29. Imperato-McGinley, J., Sanchez, R.S., Spencer, J.R., Yee, B., and Vaughan, E.D. 1992. Comparison of the effects of the 5 alpha-reductase inhibitor finasteride and the antiandrogen flutamide on prostate and genital differentiation: dose-response studies. Endocrinology. 131:1149-1156.

30. McIntyre, B.S., Barlow, N.J., and Foster, P.M. 2002. Male rats exposed to linuron in utero exhibit permanent changes in anogenital distance, nipple retention, and epididymal malformations that result in subsequent testicular atrophy. Toxicol. Sci. 65:62-70.

31. Welsh, M., Saunders, P.T., and Sharpe, R.M. 2007. The critical time window for androgen-dependent development of the Wolffian duct in the rat. Endocrinology. 148:3185-3195.

32. Herman, R.A., Jones, B., Mann, D.R., and Wallen, K. 2000. Timing of prenatal androgen exposure: anatomical and endocrine effects on juvenile male and female rhesus monkeys. Horm. Behav. 38:52-66.

33. Jost, A., Vigier, B., Prepin, J., and Perchellet, J.P. 1973. Studies on sex differentiation in mammals. Recent Prog. Horm. Res. 29:1-41.

34. Ogawa, S., and Nozawa, A. 1969. Effect of prenatal administration of androgen on the reproductive function in female rats. Endocrinol.Jpn. 16:599-607. 
35. Stinnakre, M.G. 1975. Period of sensitivity to androgens of the Wolff duct of the rat fetus [In French]. Arch. Anat. Microsc. Morphol. Exp. 64:45-59.

36. Wolf, C.J., Hotchkiss, A., Ostby, J.S., LeBlanc, G.A., and Gray, L.E., Jr. 2002. Effects of prenatal testosterone propionate on the sexual development of male and female rats: a dose-response study. Toxicol. Sci. 65:71-86

37. Neri, R., Florance, K., Koziol, P., and Van Cleave, S. 1972. A biological profile of a nonsteroidal antiandrogen, SCH 13521 (4'-nitro-3'trifluoromethylisobutyranilide). Endocrinology. 91:427-437.

38. Anonymous. 2008. Eulexin: clinical pharmacology. RxList: the Internet drug index. http://www.rxlist. $\mathrm{com} / \mathrm{cgi} /$ generic/flutam_cp.htm.

39. Salazar-Martinez, E., Romano-Riquer, P., YanezMarquez, E., Longnecker, M.P., and HernandezAvila, M. 2004. Anogenital distance in human male and female newborns: a descriptive, cross-sectional study. Environ. Health. 3:8.

40. Hughes, I.A. 1998. Congenital adrenal hyperplasia - a continuum of disorders. Lancet. 352:752-754.

41. McIntyre, B.S., Barlow, N.J., and Foster, P.M. 2001. Androgen-mediated development in male rat offspring exposed to flutamide in utero: permanence and correlation of early postnatal changes in anogenital distance and nipple retention with malformations in androgen-dependent tissues. Toxicol. Sci. 62:236-249.

42. Mylchreest, E., Sar, M., Cattley, R.C., and Foster, P.M. 1999. Disruption of androgen-regulated male reproductive development by di(n-butyl) phthalate during late gestation in rats is different from flutamide. Toxicol. Appl. Pharmacol. 156:81-95.
43. Foster, P.M., and Harris, M.W. 2005. Changes in androgen-mediated reproductive development in male rat offspring following exposure to a single oral dose of flutamide at different gestational ages. Toxicol. Sci. 85:1024-1032.

44. Spencer, J.R., Torrado, T., Sanchez, R.S., Vaughan, E.D., Jr., and Imperato-McGinley, J. 1991. Effects of flutamide and finasteride on rat testicular descent. Endocrinology. 129:741-748.

45. Baskin, L.S., et al. 1997. The effect of testosterone on androgen receptors and human penile growth. J. Urol. 158:1113-1118.

46. Sajjad, Y., Quenby, S., Nickson, P., Lewis-Jones, D.I., and Vince, G. 2004. Immunohistochemical localization of androgen receptors in the urogenital tracts of human embryos. Reproduction. 128:331-339.

47. Murakami, R. 1987. A histological study of the development of the penis of wild-type and androgen-insensitive mice. J. Anat. 153:223-231.

48. Boas, M., et al. 2006. Postnatal penile length and growth rate correlate to serum testosterone levels: a longitudinal study of 1962 normal boys. Eur. J. Endocrinol. 154:125-129.

49. Sinnecker, G.H., Hiort, O., Nitsche, E.M., Holterhus, P.M., and Kruse, K. 1997. Functional assessment and clinical classification of androgen sensitivity in patients with mutations of the androgen receptor gene. German Collaborative Intersex Study Group. Eur. J. Pediatr. 156:7-14.

50. Gorski, R.A. 2002. Hypothalamic imprinting by gonadal steroid hormones. Adv. Exp. Med. Biol. 511:57-70; discussion 70-73.

51. Knickmeyer, R.C., and Baron-Cohen, S. 2006. Fetal testosterone and sex differences in typical social development and in autism. J. Child Neurol. 21:825-845.

52. Perakis, A., and Stylianopoulou, F. 1986. Effects of a prenatal androgen peak on rat brain sexual differentiation. J. Endocrinol. 108:281-285.

53. Bowman, C.J., Barlow, N.J., Turner, K.J., Wallace, D.G., and Foster, P.M. 2003. Effects of in utero exposure to finasteride on androgen-dependent reproductive development in the male rat. Toxicol. Sci. 74:393-406.

54. Swan, S.H., et al. 2005. Decrease in anogenital distance among male infants with prenatal phthalate exposure. Environ. Health Perspect. 113:1056-1061.

55. Foster, P.M., Mylchreest, E., Gaido, K.W., and Sar, M. 2001. Effects of phthalate esters on the developing reproductive tract of male rats. Hum. Reprod. Update. 7:231-235.

56. Mylchreest, E., Cattley, R.C., and Foster, P.M. 1998. Male reproductive tract malformations in rats following gestational and lactational exposure to $\mathrm{Di}(\mathrm{n}$ butyl) phthalate: an antiandrogenic mechanism? Toxicol. Sci. 43:47-60.

57. Mylchreest, E., Wallace, D.G., Cattley, R.C., and Foster, P.M. 2000. Dose-dependent alterations in androgen-regulated male reproductive development in rats exposed to Di(n-butyl) phthalate during late gestation. Toxicol. Sci. 55:143-151.

58. Scott, H.M., et al. 2007. Role of androgens in fetal testis development and dysgenesis. Endocrinology. 148:2027-2036.

59. Hsieh, M.H., Breyer, B.N., Eisenberg, M.L., and Baskin, L.S. 2008. Associations among hypospadias, cryptorchidism, anogenital distance, and endocrine disruption. Curr. Urol. Rep. In press. 